\title{
Comparison of the effect of exercise on stable
} surface versus unstable surface on cross section area of lumbar multifidus muscle and thickness of abdominal muscle in patients with chronic low back pain

\section{Mozhgan Emami}

Shiraz University of Medical Sciences

\section{Farahnaz Emami}

Shiraz University of Medical Sciences

\section{Farahnaz Ghafari Nezhad}

Shiraz University of Medical Sciences

Shohreh Taghizadeh ( $\nabla$ taghizsh@sums.ac.ir)

Shiraz University of Medical Sciences

Research article

Keywords: low back pain, Core stabilization exercise, Unstable surface, Muscle thickness

Posted Date: March 12th, 2020

DOl: https://doi.org/10.21203/rs.3.rs-16868/v1

License: (9) This work is licensed under a Creative Commons Attribution 4.0 International License. Read Full License 


\section{Abstract}

Objective

The aims of this study were, comparison the effects of core stability exercises performed on a stable or unstable surface on the thickness of abdominal and lumbar multifidus muscle in patients with nonspecific chronic low back pain.

Method

Thirty-four patients were randomly assigned to an unstable or stable surface group ( $n=17$ each). Both groups did five core stability exercises for 6 weeks (three times per week). the thickness of the core muscle was evaluated pre and post-treatment.

Results

After 6 weeks of training, in both groups, all variables improve. internal oblique muscle thickness and transversus abdominis thickness in the unstable surface group improved significantly more than those in the stable surface group $(p<0.05)$.

Conclusion

Our findings indicate exercise on an unstable surface more effective in improving the core muscle thickness relative to stable surface in patients with non-specific chronic low back pain. However, extra studies are required to support our findings.

\section{Background}

Low back pain (LBP) is described as ache between the twelfth rib and lower buttock fold with or without lower extremity pain(1). The second most current cause for seeing a clinician, and one of leading cause for absenteeism from work is related to the musculoskeletal system disorder; particularly low back pain(2). LBP is a multifactorial and widespread disorder affecting both industrialized and nonindustrialized countries(3-5) and nearly $80 \%$ of people experience it at some day in their lives(6). The economic burden of LBP to society included indirect costs (work days lost) and direct costs (treatment costs)(7).

Chronic LBP (CLBP) is usually described symptoms that lasting further than 3 months $(1,2)$. Non-specific LBP is determined as back pain without identifiable pathology. Most LBP is non-specific, but $5-10 \%$ of cases have specific cause. possible sources of pain in "non-specific CLBP" are some degenerative conditions, metabolic bone disease, psychogenic pain, inflammatory conditions, infectious causes, trauma, referred pain and congenital disorders $(8,9)$. 
According to European Guidelines the first stage treatment for Management of Non-Specific CLBP is exercise therapy(3). The core has been define as a muscular girdle to stabilize the trunk and spine, with and without limb movement. the aim of core Stability exercise is training and coordination of abdominal and lumbopelvic region $(3,10)$. In healthy subjects in order to stabilize the lumbar spine, the transversus abdominis and multifidi are the first muscles to be contracted before shoulder and leg movement $(4,10)$. But in patients with LBP contraction of these muscles was significantly delayed(11).

Ultrasound imaging is an objective and non-invasive method for measuring muscle morphology, crosssectional areas (CSA) and muscle thicknesses $(4,12)$. In addition, ultrasound has been used for evaluation of muscle atrophy and hypertrophy as well as for assessment of the effectiveness of the rehabilitation programs(4).

I.R. Scott et al. reported significant increases in CSA of LM when sitting on an unstable surface compared to a stable surface in both healthy subjects and patients with $\operatorname{CLBP}(5)$. It has been assumed that performing training on unstable conditions provides higher stress to the entire musculature(13). Previous studies noted that an unstable surface stimulated the sensory and motor system further than a stable surface(14).

The objective of this research was to compare trunk muscle thickness under stable and unstable conditions in patients with chronic non-specific LBP. Based on previous study, our supposed was that when performing training on an unstable surface trunk muscle thickness more increase.

\section{Methods}

\section{Study Design}

This study was single-blind randomized clinical trial and conducted between July 2019 and December 2019. This study was approved by the Ethics Committees of Shiraz University of Medical Science in date 2019-02-27 and registered in the Iranian Registry of Clinical Trials (IRCT) as IRCT20190527043730N1. all participants were conscious of their treatment but were blinded to the sort of treatment in the other group. Subjects: The sample size was determined via the difference between two independent means (two groups: $t$ test-means) formula. Thirty-four Patients with non-specific CLBP were recruited to the Faculty of Rehabilitation Sciences of Shiraz University of Medical Sciences through printed advertisements on notice boards at various clinics. these patients randomly allocated into two experimental groups equally: group A carried out core stability training on a stable surface and group B carried out them on an unstable surface. The participants were randomized via permuted block randomization method with 2 block size.

The inclusion criteria consisted of: Patients with diagnosed mechanical low back pain, both males and females, age of 18-40 years, LBP with an onset duration $>3$ months, pain intensity of 3-8 in the VAS Patients with minimum to moderate disability on ODI(15). The exclusion criteria consisted of: patients with pregnancy, spinal fractures, herniated discs, acute back pain, systemic disc herniation, severe 
osteoarthritis, spondylolisthesis and spondylolysis, lower limb length discrepancy, previous surgery on vertebral column, malignancy, scoliosis(15-17).

\section{Outcome measurements}

Before and after the treatment, data on the thickness of the transverse abdominis (TrA), internal oblique (IO), external oblique(EO) and lumbar multifidus(LM), as well as cross-section area (CSA) of LM were collected using ultrasound Imaging.

\section{Thickness of the lateral abdominal muscles}

Bilateral thickness of the EO, $\mathrm{IO}$ and TrA muscles were measured using a HS-2600 diagnostic ultrasound imaging unit (Honda Electronics Inc., Honda, Japan) that was set in B-mode and used a head transducer with $7.5 \mathrm{MHz}, 60-\mathrm{mm}$, board band linear array (18). All thickness were measured at the relaxation and during the abdominal drawing-in maneuver (ADIM). The lateral abdominal muscles measurement was performed with the Participant in the supine crook-lying position and the transducer placed transversely at the center point of the anterior axillary line (the midpoint amid the iliac crest and the last rib). In the supine crook-lying position, a Pressure Biofeedback Unit (PBU, Chattanooga Group, Hixson, TN, USA) was located below the participant's lumbar spine and the bulb was filled with the air up to a pressure of $40 \mathrm{mmHg}(18-20)$. Then, the abdominal muscle activation measurements were recorded while the participants performed the ADIM. In this maneuver participants gently pull their lower abdominal muscles in toward their spine While pressure applied to the PBU was increased 0, 2 or $4 \mathrm{mmHg}$. contraction held for $7 \mathrm{~s}$. all image was stored at the end of expiration.

\section{Thickness of the lumbar multifidus}

Longitudinal view of bilateral LM muscle at the L4-5 level was obtained at rest and during a contralateral arm lift with the participant positioned prone. In order to reduce the lumbar curve used a cushion below the abdomen and the hip. The transducer was located longitudinally along the L4 spinous process, shifted laterally, and then slightly was angled in a medial direction. This point was directly over the L4/L5 facet joint. The space between this landmark and the plane between the muscle and subcutaneous tissue was used to the thickness measurement of LM.

\section{Cross-section area of the lumbar multifidus}

To determine the CSA of LM, in first the transducer was placed longitudinally on the L4 spinous process, then was rotated 90 degrees to put transversely in the midline. The echogenic vertebral laminae were used as a landmark to recognized the inferior border of the LM muscle. The multifidus muscle is bordered medially by the acoustic shadow from the tip of the spinous process of the vertebral, and the superior border was determined by the thoracolumbar fascia. The lateral border was provided by the fascia separating multifidus muscle from the longissimus. The contralateral upper extremity lifting task was performed with $120^{\circ}$ shoulder abduction and $90^{\circ}$ elbow flexion. participant lifted a light hand weight off the table nearly $5 \mathrm{~cm}$ and held it for $8 \mathrm{~s}$. 


\section{Intervention and procedure}

In this study, subjects who satisfied the selection criteria were randomly divided into the stable surface group (group A) and unstable surface group (group B).

During the study period, all subjects completed general physical therapy, consisting of a 20-minute hot pack treatment, 20-minute Conventional TENS, characterized by continuous stimulation at high frequencies $(80-100 \mathrm{~Hz})$, with wave durations of 50-100 ms and low intensities, and 5-minute ultrasonic treatment $(0.8-1 \mathrm{MHz})(21)$. After patients had undergone general physical therapy, they had performed the lumbar stabilization exercise program. The lumbar stabilization exercise program was consist of elbow-toe, back bridge, side bridge, hand-knee, and curl-up motions.

Both the groups were asked to carry out 5 types of core stability exercises thrice a week for 6 weeks (total of 18 sessions)(22). The intensity of the exercise program was advanced every 2 weeks.

Curl-up: The participant was on the supine position with knees bent and feet flat on the ground. Hands were clasped in back of the head. The subject Slowly lifting the thoracic spine so that the scapulae were off the floor with keeping abdominal muscles draw in. The subjects performed the curl-up both on the floor (stable status) and with a balance cushion (unstable status).

Pelvic bridging: The participant was lying in the supine position with arms out to the side, feet flat on the ground, knees flexed at $90^{\circ}$ and toes facing forward. The participant lifted the pelvis to degree hip flexion get zero. The participant carried out the pelvic bridge position on the ground (stable status) and with the feet and trunk on the balance cushion (unstable status).

Prone plank: The participant assumes a prone position on the ground with elbows were below the shoulders and the brachiums were vertical to the ground, while only the toes and forearms were palpating the ground. The participant carried out the prone plank motion on the ground (stable status) and with the forearms and toes on a balance cushion (unstable status).

Side plank: The participant assumes a side plank position with elbow below the shoulder and brachiums vertical to the floor. The participant lift the pelvis off the ground such that that the body aligned in a straight line, supporting points were on elbow and feet. Subjects carried out the side plank on the ground (stable status) and with the elbow and feet on the balance cushion (unstable status).

Bird-Dog: The participants were placed in a quadruped position and were requested them to raise the upper limb directly, whilst concurrently raising the opposite lower limb as well as keep it direct. In this position, participants held their pelvis in a neutral position and also were breathing as normal. Participants performed the bird-dog on the floor (stable status) and with a balance cushion (unstable status).(16, 17, 23).

\section{Data analysis}


For the data analysis, SPSS version 23 software was used. The normality of all variables was performed by Shapiro-Wilk test. The demographic characteristics (age, height, weight, pain intensity and oswestry disability index) were not significant differences between the two groups $(P>0.05)$ (Table1). The independent t-test and Mann-Whitney $\mathrm{U}$ test were used to compare the size of the muscles, before and after the trunk stabilization exercises, between the two experimental groups for parametric and nonparametric data, respectively. The paired t-test and Wilcoxon test were used to analyze the muscle size in each group, before and after the trunk stabilization exercises for parametric and nonparametric data, respectively. The statistical significance level was accepted A p-value lower than 0.05 .

\section{Results}

Comparison of the before and after results showed that the thickness of the left and right external oblique during rest and contraction, thickness of the left internal oblique during contraction and thickness of the left TrA during rest and contraction significantly increased in group $A(p<0.05)$. In group $B$, thickness of the left and right all abdominal mucsles (TrA, IO, EO) during rest and contraction, thickness of the left multifidus muscle during rest and contraction and thickness of the right multifidus muscle during contraction significantly increased $(p<0.05)$. The cross-sectional areas of the left and right multifidus muscles during rest and contraction significantly increased in both group $(p<0.05)$.

Comparison of changes between the two groups revealed that the resting thickness of the left internal oblique, resting thickness of the left TrA, resting and contraction thickness of the left multifidus muscle, resting and contraction thickness of the right internal oblique, resting and contraction thickness of the right TrA and cross-sectional area of left multifidus muscle during rest and contraction in group $B$ greater than those in the group $A(p<0.05)$. Other variables showed no significant difference between the two groups $(p>0.05)$.

Table 1

Participants' Demographic information

\begin{tabular}{|c|c|c|c|}
\hline $\begin{array}{l}\text { Group } \\
\text { variable }\end{array}$ & Stable surface $(n=17)$ & Unstable surface $(n=17)$ & $P$ value \\
\hline \multirow[t]{2}{*}{ Gender } & 8 & 6 & \multirow[t]{2}{*}{$0 / 56$} \\
\hline & 9 & 11 & \\
\hline Age (yrs.) & $31 / 47$ & $29 / 35$ & $0 / 322$ \\
\hline $\mathrm{BMI}(\mathrm{Kg} / \mathrm{m} 2)$ & $25 / 08$ & $24 / 98$ & $0 / 973$ \\
\hline VAS & 6 & $5 / 53$ & $1 / 00$ \\
\hline ODI & 32 & 30 & $0 / 357$ \\
\hline \multicolumn{4}{|c|}{ BMI: body mass index, VAS: Visual Analogue Scale, ODI: Oswestry Disability Index } \\
\hline
\end{tabular}




\section{Discussion}

This study examined whether core stability exercises performed on an unstable surface were more effective at improving abdominal muscle thickness and thickness and cross-section area of lumbar multifidus muscle rather than stable surface during 18 session treatment.

After the 18 sessions, lumbar stabilization exercise, group A showed significant improvements in thickness of the left and right external oblique during rest and contraction, thickness of the left internal oblique during contraction, thickness of the left TrA during rest and contraction and CSA of left and right lumbar multifidus during rest and contraction. But other variables not improved in this group. However, the unstable surface group showed significant difference in all variables except resting thickness of right lumbar multifidus. Between the post-treatment values, the unstable surface group demonstrated significant improvements in resting thickness of the left internal oblique, resting thickness of the left TrA, resting and contraction thickness of the left multifidus muscle, resting and contraction thickness of the right internal oblique, resting and contraction thickness of the right TrA and CSA of left multifidus muscle during rest and contraction compared with the stable surface group.

usually, hypertrophy in Muscle happens 8 to 12 weeks after strength training, therefore the short period of the current study and the effects of neuromuscular adaptation may have caused changes in several variables $(24,25)$.

Numerous studies have shown a decrease in the thickness of TrA, internal oblique and lumbar multifidus muscles at rest and contraction in patients with chronic low back pain. Biderman et al also found that lumbar multifidus muscles had more fatiguability in patient with chronic low back pain relative to other parts of the erector spine(26). Kliziene et al. In 2015 and Hides et al. In 2008 reported core stabilization exercises in patients with chronic low back pain increase cross-section area of the lumbar multifidus muscle(27). Similar studies have been conducted regarding the effect of core stability exercises on an unstable surface on muscle thickness. Marshall et al., 2006, showed hypertrophy of lumbar multifidus muscles in patients with chronic low back pain after a 12-weeks period of exercise on the swiss ball(28).

In contrast with our study, Danneels et al. and Akbari et al. demonstrated positive results for increased CSA of lumbar multifidus in favor of the general exercise. On the other hand, they believe that stabilization exercises do not provide additional benefit to patients with chronic LBP(26).

The main feature of the core stability exercise is the training of the deep trunk muscles and coordination of the deep and the superficial trunk muscles(26). Rasouli et al. (2011) noted in patients with CLBP, thickness of TrA increased as stability decreased(5). Kim et al. reported that training on an unstable surface improved trunk asymmetry. Also, Caroline reported that exercise on an unstable surface increased proprioception in trunk muscles(12). As a result, many investigators claim training under unstable conditions improve joint position sense, balance, posture and proprioception that focus on muscle recruitment, and also provides a greater stress on the core musculature $(29,30)$. 


\section{Conclusion}

This study provides evidence of increased thickness of internal oblique, $\operatorname{Tr} A$, and lumbar multifidus muscles when training on an unstable surface compared to a firm surface in individuals with CLBP. These findings supported the use of an unstable surface in rehabilitation to improved trunk muscle size.

\section{Abbreviations}

LM: Lumbar Multifidus, CLBP: Chronic Low Back Pain, CSA: Cross-Sectional Area, IRCT: Iranian Registry of Clinical Trial, VAS: Visual Analogue Scale, ODI: Oswestry Disability Index, TrA: Transverse Abdominis, IO: Internal Oblique, EO: External Oblique, ADIM: Abdominal Drawing-In Maneuver, TENS: Transcutaneous Electrical Nerve Stimulation

\section{Declarations}

\section{Ethics approval and consent to participate}

This study was approved by the Ethics Committees of Shiraz University of Medical Science in date 201902-27. participants were provided exact information about the study process and safety and they were allowed to exclude from the study without revealing any reason at any time. All patients signed informed consent.

\section{Consent for publication}

Not applicable.

\section{Availability of data and material}

The raw data sets used and/or analyzed in this study are available from the corresponding author on reasonable request.

\section{Competing interests}

The authors declare that they have no conflicts of interest.

\section{Funding}

This study did not receive any external funding.

\section{Authors' contributions}

ME participated in the data collection, data analysis and writing of the manuscript; FGN participated in the data collection, data analysis and writing of the manuscript; FE participated in the data analysis and interpretation of data; ST participated in the design, data analysis and revisions of the manuscript. 


\section{Acknowledgements}

We are grateful to all the patients participated in this study.

\section{References}

1. Pourahmadi MR, Ebrahimi Takamjani I, Jaberzadeh S, Sarrafzadeh J, Sanjari MA, Mohsenifar H, et al. The Effect of Core Stabilization Exercise on the Kinematics and Joint Coordination of the Lumbar Spine and Hip During Sit-to-Stand and Stand-to-Sit in Patients With Chronic Nonspecific Low Back Pain (COSCIOUS): Study Protocol for a Randomized Double-Blind Controlled Trial. JMIR research protocols. 2017;6(6):e109.

2. Franca FR, Burke TN, Hanada ES, Marques AP. Segmental stabilization and muscular strengthening in chronic low back pain: a comparative study. Clinics. 2010;65(10):1013-7.

3. Gomes-Neto M, Lopes JM, Conceicao CS, Araujo A, Brasileiro A, Sousa C, et al. Stabilization exercise compared to general exercises or manual therapy for the management of low back pain: $A$ systematic review and meta-analysis. Physical therapy in sport : official journal of the Association of Chartered Physiotherapists in Sports Medicine. 2017;23:136-42.

4. Ghamkhar L, Emami M, Mohseni-Bandpei MA, Behtash H. Application of rehabilitative ultrasound in the assessment of low back pain: a literature review. Journal of bodywork and movement therapies. 2011;15(4):465-77.

5. Scott IR, Vaughan AR, Hall J. Swiss ball enhances lumbar multifidus activity in chronic low back pain. Physical therapy in sport : official journal of the Association of Chartered Physiotherapists in Sports Medicine. 2015;16(1):40-4.

6. Wang X-Q, Zheng J-J, Yu Z-W, Bi X, Lou S-J, Liu J, et al. A meta-analysis of core stability exercise versus general exercise for chronic low back pain. PloS one. 2012;7(12):e52082.

7. Krismer M, Van Tulder M. Low back pain (non-specific). Best practice \& research clinical rheumatology. 2007;21(1):77-91.

8. Behennah J, Conway R, Fisher J, Osborne N, Steele J. The relationship between balance performance, lumbar extension strength, trunk extension endurance, and pain in participants with chronic low back pain, and those without. Clinical biomechanics. 2018;53:22-30.

9. Balagué F, Mannion AF, Pellisé F, Cedraschi C. Non-specific low back pain. The Lancet. 2012;379(9814):482-91.

10. Akuthota V, Ferreiro A, Moore T, Fredericson M. Core stability exercise principles. Current sports medicine reports. 2008;7(1):39-44.

11. Hodges PW, Richardson CA. Inefficient muscular stabilization of the lumbar spine associated with low back pain: a motor control evaluation of transversus abdominis. Spine. 1996;21(22):2640-50.

12. Yoo J, Jeong J, Lee W. The effect of trunk stabilization exercise using an unstable surface on the abdominal muscle structure and balance of stroke patients. Journal of physical therapy science. 2014;26(6):857-9. 
13. Anderson KG, Behm DG. Maintenance of EMG activity and loss of force output with instability. The Journal of Strength \& Conditioning Research. 2004;18(3):637-40.

14. Bae SH, Lee HG, Kim YE, Kim GY, Jung HW, Kim KY. Effects of trunk stabilization exercises on different support surfaces on the cross-sectional area of the trunk muscles and balance ability. Journal of physical therapy science. 2013;25(6):741-5.

15. Kamali F, Zamanlou M, Ghanbari A, Alipour A, Bervis S. Comparison of manipulation and stabilization exercises in patients with sacroiliac joint dysfunction patients: A randomized clinical trial. Journal of bodywork and movement therapies. 2019;23(1):177-82.

16. Kang TW, Lee JH, Park DH, Cynn HS. Effect of 6-week lumbar stabilization exercise performed on stable versus unstable surfaces in automobile assembly workers with mechanical chronic low back pain. Work. 2018;60(3):445-54.

17. Balakrishnan R, Yazid E, Mahat MFB. Effectiveness of the core stabilisation exercise on floor and Swiss ball on individual with non-Specific low back pain. International Journal of Physical Education, Sports and Health. 2016;3(1):347-56.

18. Emami F, Yoosefinejad AK, Razeghi M. Correlations between core muscle geometry, pain intensity, functional disability and postural balance in patients with nonspecific mechanical low back pain. Medical engineering \& physics. 2018;60:39-46.

19. Park D-J, Lee S-K. What is a suitable pressure for the abdominal drawing-in maneuver in the supine position using a pressure biofeedback unit? Journal of physical therapy science. 2013;25(5):527-30.

20. Kiesel KB, Underwood FB, Mattacola CG, Nitz AJ, Malone TR. A comparison of select trunk muscle thickness change between subjects with low back pain classified in the treatment-based classification system and asymptomatic controls. The Journal of orthopaedic and sports physical therapy. 2007;37(10):596-607.

21. Buchmuller A, Navez M, Milletre-Bernardin M, Pouplin S, Presles E, Lanteri-Minet M, et al. Value of TENS for relief of chronic low back pain with or without radicular pain. European journal of pain. 2012;16(5):656-65.

22. Marshall PW, Murphy BA. Evaluation of functional and neuromuscular changes after exercise rehabilitation for low back pain using a Swiss ball: a pilot study. Journal of manipulative and physiological therapeutics. 2006;29(7):550-60.

23. Imai A, Kaneoka K, Okubo Y, Shiina I, Tatsumura M, Izumi S, et al. Trunk muscle activity during lumbar stabilization exercises on both a stable and unstable surface. The Journal of orthopaedic and sports physical therapy. 2010;40(6):369-75.

24. Hosseinifar M, Akbari M, Behtash H, Amiri M, Sarrafzadeh J. The effects of stabilization and McKenzie exercises on transverse abdominis and multifidus muscle thickness, pain, and disability: a randomized controlled trial in nonspecific chronic low back pain. Journal of physical therapy science. 2013;25(12):1541-5.

25. Shamsi M, Sarrafzadeh J, Jamshidi A, Zarabi V, Pourahmadi MR. The effect of core stability and general exercise on abdominal muscle thickness in non-specific chronic low back pain using 
ultrasound imaging. Physiotherapy theory and practice. 2016;32(4):277-83.

26. Akbari A, Khorashadizadeh S, Abdi G. The effect of motor control exercise versus general exercise on lumbar local stabilizing muscles thickness: randomized controlled trial of patients with chronic low back pain. Journal of back and musculoskeletal rehabilitation. 2008;21(2):105-12.

27. Kliziene I, Sipaviciene S, Klizas S, Imbrasiene D. Effects of core stability exercises on multifidus muscles in healthy women and women with chronic low-back pain. Journal of back and musculoskeletal rehabilitation. 2015;28(4):841-7.

28. Marshall PW, Murphy BA. Core stability exercises on and off a Swiss ball. Archives of physical medicine and rehabilitation. 2005;86(2):242-9.

29. Rasouli O, Arab AM, Amiri M, Jaberzadeh S. Ultrasound measurement of deep abdominal muscle activity in sitting positions with different stability levels in subjects with and without chronic low back pain. Manual therapy. 2011;16(4):388-93.

30. Anderson K, Behm DG. Trunk muscle activity increases with unstable squat movements. Canadian journal of applied physiology. 2005;30(1):33-45.

\section{Figures}




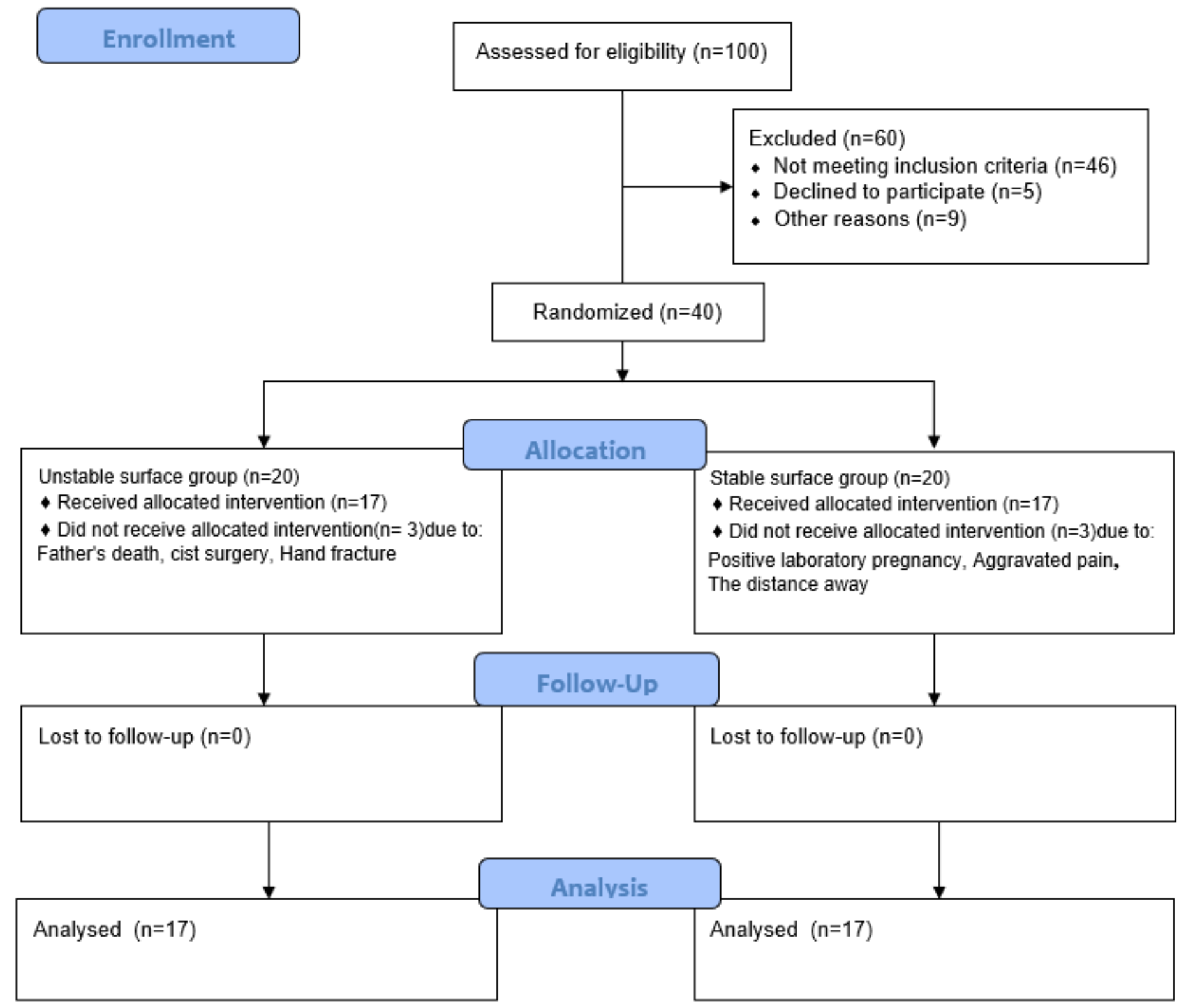

\section{Figure 1}

CONSORT flowchart 